\title{
Efficient 2-D Structuring Element for Noise Removal of Grayscale Images using Morphological Operations
}

\author{
Mangala A. G. \\ Department of Master of \\ Computer Application, \\ N.M.A.M. Institute of Technology, Nitte.
}

\author{
Balasubramani R., PhD \\ Department of Information \\ Science Engineering \\ N.M.A.M. Institute of Technology, Nitte.
}

\begin{abstract}
Mathematical morphological (MM) operations plays vital role in image processing and in enhancing the region of shape. Especially the application of basic morphological operations is used in enhancing the image quality.

This paper describes an experiment with morphological operations for reducing the salt-and- pepper noise from the images of bacteria. The quality of enhanced images is measured based on image quality assessment operations. Experiment's results are given in comparison with various 2Dflat Structuring Element(SE).
\end{abstract}

\section{General Terms}

Image Processing, Pattern Recognition.

\section{Keywords}

Mathematical morphology, Structuring element, Lactococcus.

\section{INTRODUCTION}

Mathematical morphology is based on set theory principles. This includes techniques to process digital images. Morphological operations works on geometrical shape of the object in an image. A morphological operation considers relatively ordered pixel values. Thus morphological operations are mainly suitable for gray scale or binary images. Previous experiments [12] [13] [14] [1] [2] shows that MM operations have been used to subjectively reduce the noise level in images.

Random variation in image brightness is noise. Noise degrades an image. Primarily noise is added to the image due to image acquisition phase in which an optical image converted to continuous electrical signal and then it is sampled [14]. Noise removal without image distortion is almost impossible. But noise must be reduced to certain acceptable level to analyze the image for further processing. Salt-and-pepper noise is a type of additive noise which is most common in images. Malfunctioning camera sensors, image signal transmission in noisy channel, corrupted memory areas, timing faults in the digitization step may result in salt-andpepper noise [14].

\section{MORPHOLOGICAL OPERATIONS}

In image processing morphological operations highly experimented [6] in improving the appearance. To reduce the noise the MM is also applied it uses structuring element to probe the image and thereby useful information from the image can be obtained and noise can be reduced while preserving the features. This paper is on an experiment in which four morphological operations are working to reduce the noise from the gray scale image and thereby enhancing the quality of the images.

\subsection{Structuring element}

Structuring element is a simple, predefined shape, used to probe an image. Structuring element defines the neighborhood of the pixel of interest.

Especially flat structuring elements when used with morphological operations are suited to process gray scale and binary images because their light transfer function is not known and morphological operators are based only on the relative ordering of pixel values, instead of their numerical values.

From graphical pint of view, structuring elements can be represented either by a matrix having 0's and 1's or as a set of foreground pixels all having values 1 . Some conventional structuring elements like arbitrary, ball, diamond. There are two types of structuring elements, flat SE and non flat SE [15]. In this paper five arbitrary flat structuring elements namely disk, square, rectangle, line, octagon has been used for the experiments and shown in below.

$\left.\begin{array}{lllll}\hline 1 & 1 & 1 & 1 & 1 \\ 1 & 0 & 0 & 0 & 1 \\ 1 & 0 & 0 & 0 & 1 \\ 1 & 0 & 0 & 0 & 1 \\ 1 & 1 & 1 & 1 & 1\end{array}\right]\left[\begin{array}{lllllll|}1 & 1 & 1 & 1 & 1 & 1 & 1 \\ 1 & 0 & 0 & 0 & 0 & 0 & 1 \\ 1 & 0 & 0 & 0 & 0 & 0 & 1 \\ 1 & 0 & 0 & 0 & 0 & 0 & 1 \\ 1 & 1 & 1 & 1 & 1 & 1 & 1\end{array}\right.$

Fig 1: Square Boundary Fig 2: Rectangle Boundary

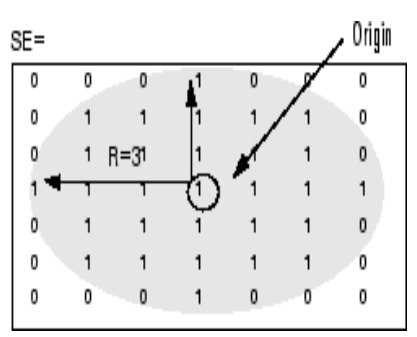

Fig 3: Circle Boundary

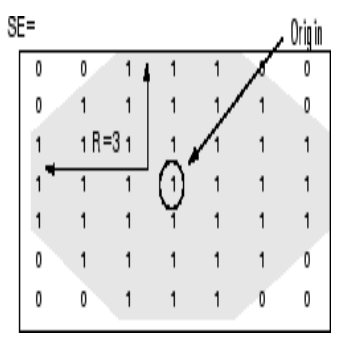

Fig 4: Octagon Boundary

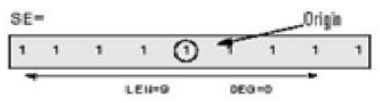

Fig 5: Line Boundary

\subsection{Dilation}

Dilation is useful in adding pixels to the region boundaries or it is also used for filling the holes in the image [7]. The problem associated in applying dilation operation is there is a chance of closing the holes completely or narrowing the holes 
in an image [9]. So dilation can stretch or shrink the original figure. Disjoint pixels can be connected or pixels can be added at edges using dilation operation.

\subsection{Erosion}

Erosion operation produces the reverse effect of dilation. In erosion boundaries will be narrowed and it expands the holes. This is done by setting an ON pixel to OFF as a structuring element sliding across the image. All the pixels which are completely overlap ON-valued pixels set to OFF.

\subsection{Opening and closing}

To obtain the desired results erosion and dilation can be applied repeatedly. However, execution order of these operations can show a difference in the processed image. Opening and closing are obtained by combining dilation and erosion.

Opening operation involves erosion followed by dilation with the same structuring element. While closing operation starts with dilation followed by erosion with the same structuring element. Smoothening the contours of objects, breaking narrow joints and eliminating thin protrusions is carried out through opening. In closing smoothing of sections of contours is possible but it fuses narrow breaks, includes gaps in the contour and removes small holes. As it is mentioned in [7], opening operation must be used if small noise regions are more in an image. Closing, on the other hand restores connectivity between close proximity objects.

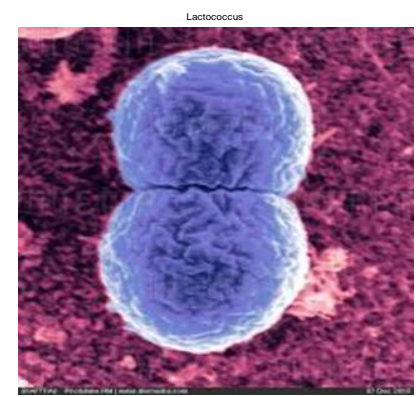

Fig 6: Original image of lactococcus

\section{PROPOSED APPROACH}

In digital image processing the term morphology refers to a special type of filtering and SEs. Selecting an appropriate SE is a highly important task in morphological image processing. SE can be represented either by a matrix having 0 's and 1's or as a set of foreground pixels all having values 1 . In both the representation, the origin of SE must be clearly identified.

This approach is based on removal of salt-and- pepper noise using morphological operations with 2D-flat structuring elements. The approach is shown diagrammatically in Figure 7. In the first stage of proposed approach color images of lactococcus bacteria in different dimensions are taken and then transformed into gray scale image in the second stage. The gray scale image is represented as intensity values varying from black at the weakest intensity to white to the strongest. In the third stage the morphological operations are executed with arbitrary flat SE which are proposed in this paper and are shown in figure 1 to 5 .

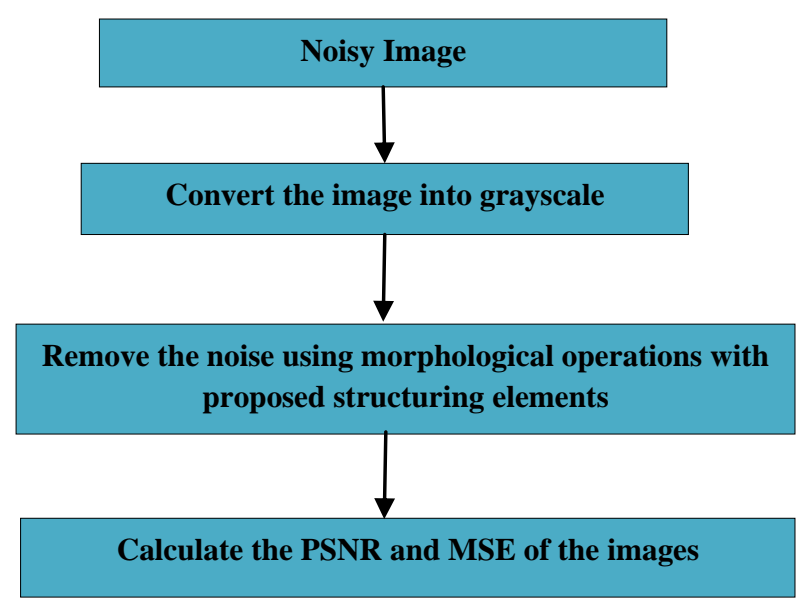

Fig 7: Steps used in the proposed approach

\section{EXPERIMENTAL RESULTS AND DISCUSSIONS}

Five flat arbitrary SE have been used to carry out the noise removal operation using morphological operators. They are disk ,square, line, octagon and rectangle, five real life images has been chosen to do the experimental work" lactococcus image of $512 * 512,460 * 819,1024 * 1024,1218 * 1120$,and 2048 * 2048 dimensions within six percent of salt and pepper noise are used. It has been observed from the final resultant images (figure 8 to figure 12) using square shaped SE most of the noise can is reduced. The statistical measurements with PSNR and MSE are also shown in fig13 to fig16.The statistical measurements of enhanced images by removing noise is shown.

It is found from the statistical measurements using the proposed approach in respective of PSNR the square boundary SE produces higher PSNR and octagon SE produces lower PSNR. In respective of MSE with octagon boundary SE produces higher MSE and square SE produces lower MSE.

\section{CONCLUSION AND FUTURE WORK}

For digital images SE plays a significant role in image enhancing by noise removal using morphological image processing. The choice of different structuring elements would lead to myriad applications and analysis and processing of geometric information of the image lied there in and thus would ultimately decide data distribution and data volume and their nature in the morphological transform. Dilate, erode open and close are the morphological operations applied on noisy image of the lactococcus bacteria in this experiment. Even if these operations have their own contributions in enhancing the images, combination of these operators are made it possible to significantly improve the appearance of the noisy image by noise reduction. The conclusions are drawn in this paper are purely based on results of the experiments.

In this paper the morphological study with five arbitrary SE to accomplish noise removal operation has been carried out. The statistical measurements are also shown and as per visual perception analysis the resultant images with square SE yields better accuracy in removing noise is recognized. In the future research, more morphological operations can be used and experimented for higher noise levels. 


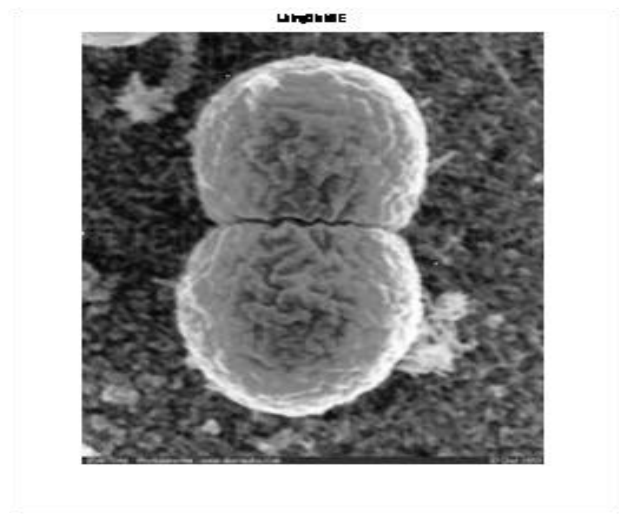

Fig 8: Using disk SE

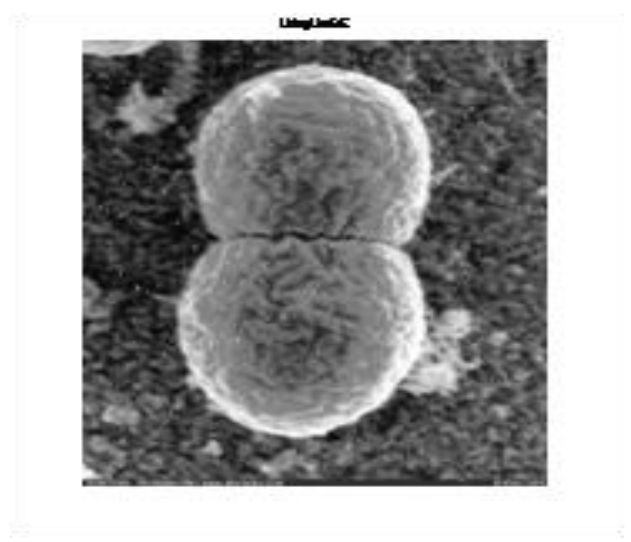

Fig 10: Using line SE

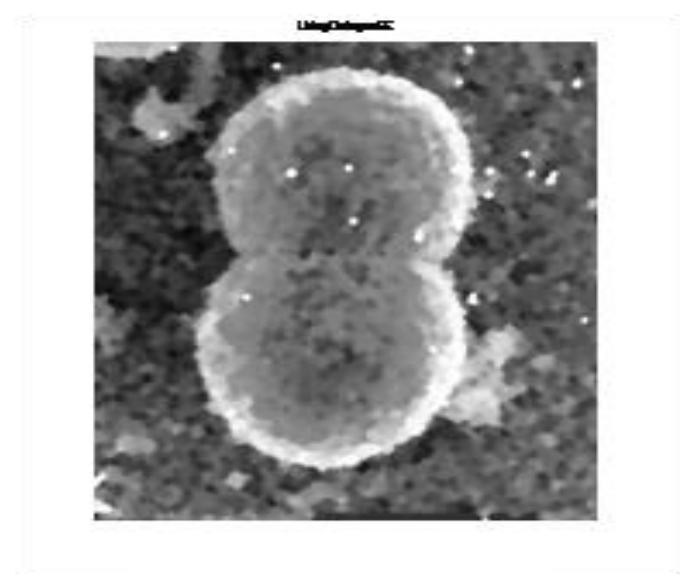

Fig 12: Using octagon SE

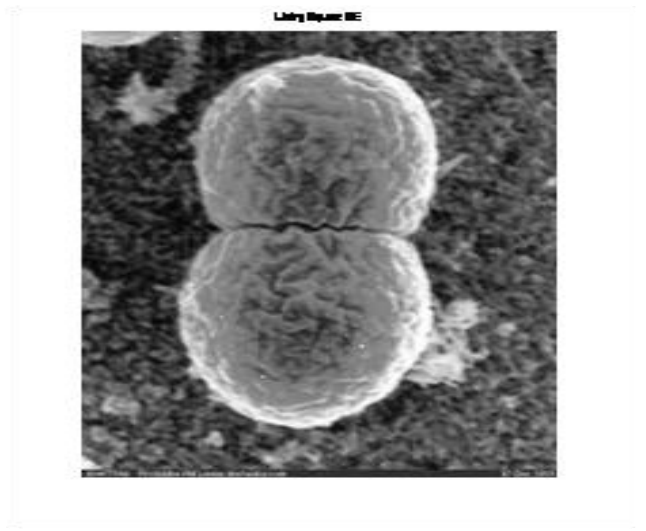

Fig 9: Using square SF

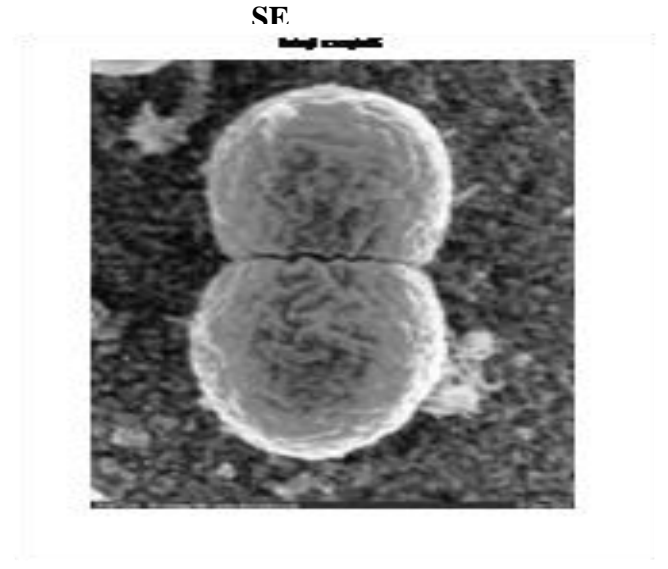

Fig 11: Using rectangle SE 


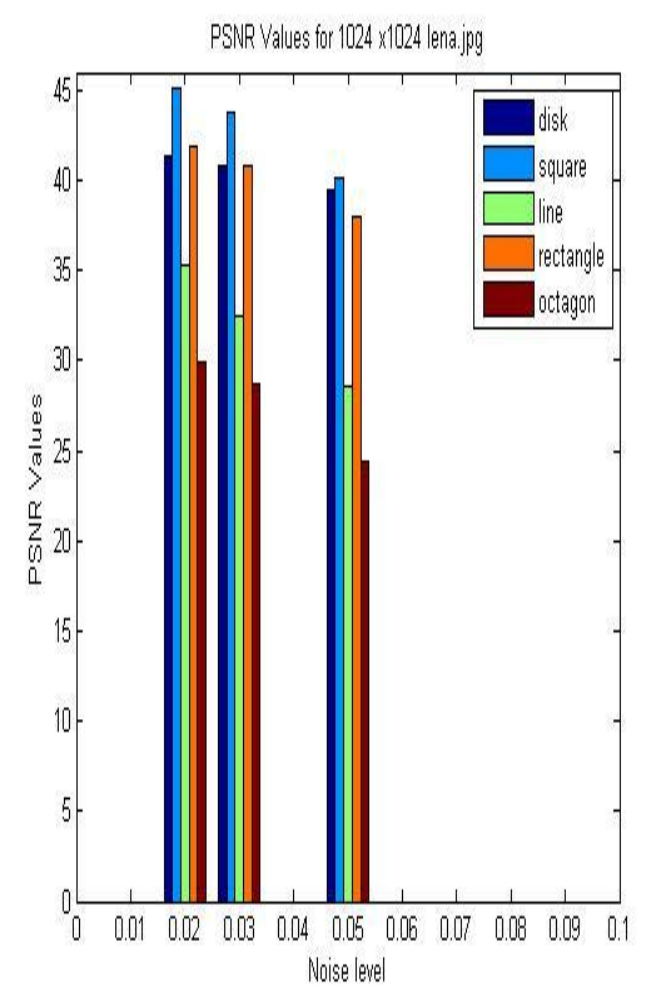

Fig 13:PSNR values for $1024 \mathrm{X} 1024$

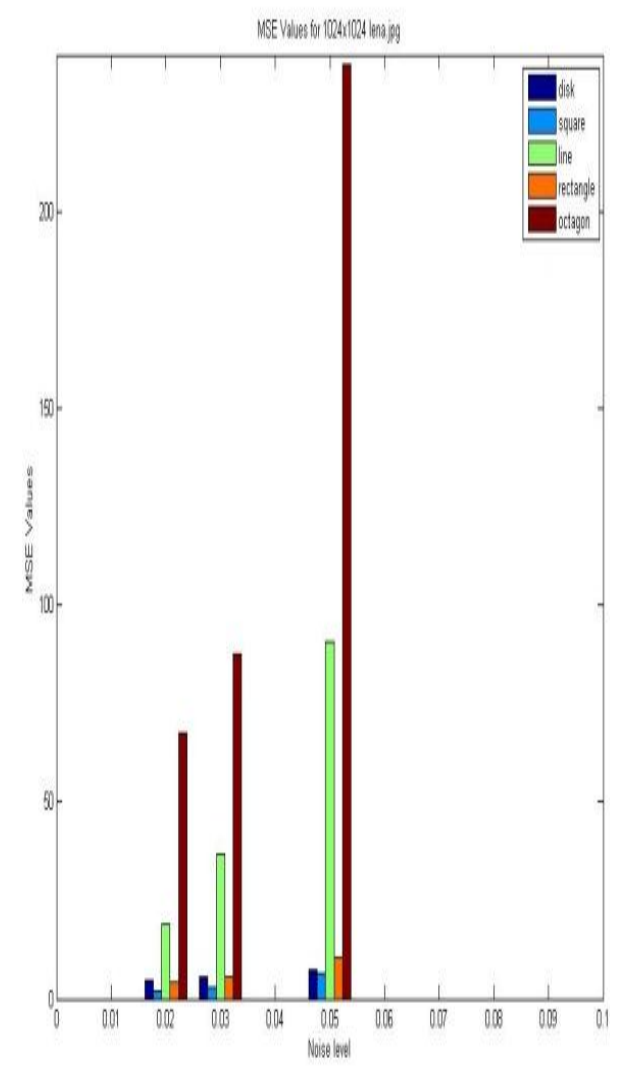

Fig 15:MSE values for $1024 \mathrm{X} 1024$ image

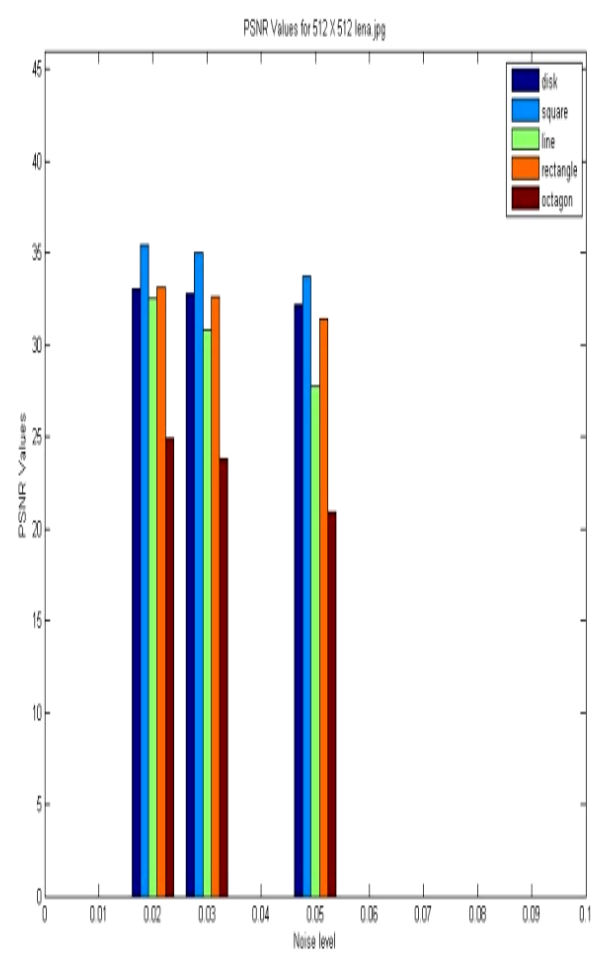

Fig 14:PSNR values for 512X512image

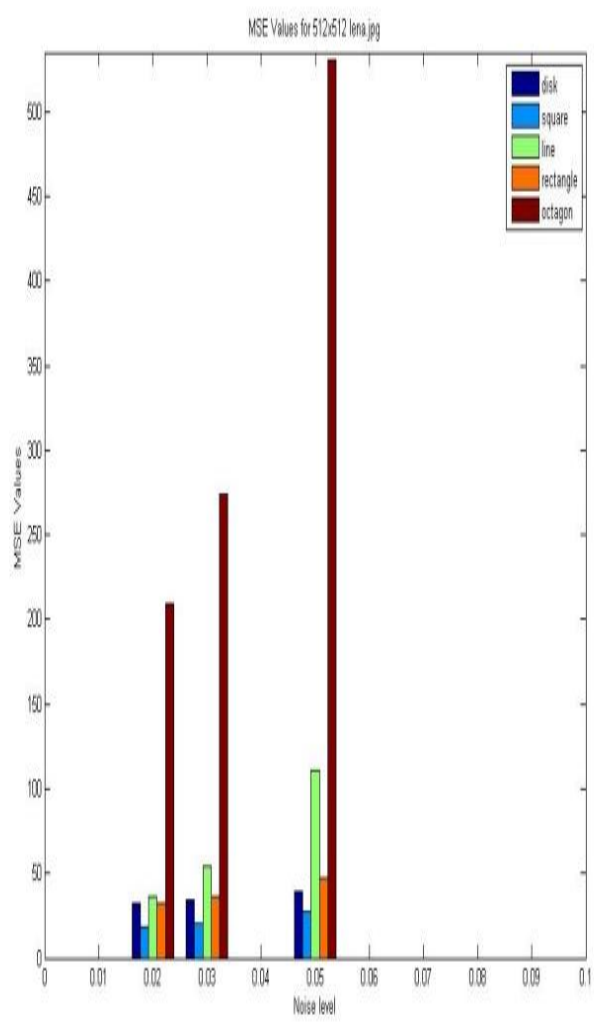

Fig 16:MSE values for 512X512image 


\section{REFERENCES}

[1] A. Taleb-Ahmed, X. Leclerc and T.S. Michel, 2001 Semi-Automatic Segmentation of Vessels by Mathematical Morphology: Application in MRI, Proceedings of International Conference on Image Processing, pp. 1063-1066.

[2] F. Ortiz, 2006 Gaussian Noise Removal by Color Morphology and Polar Color Models", Proceedings of 3rd International Conference of Image Analysis and Recognition (ICIAR '06), Portugal, , pp. 163-172.

[3] J. Song, R. L. Stevenson, and E. J. Delp, 1989 The Use of Mathematical Morphology in Image Enhancement, Proceedings of the 32nd Midwest Symposium on Circuits and Systems, Urbana-Champaign, IL, pp. 67-70.

[4] J. Zhen, M. Zhong, L. Qi and W. Qinghua, 2004 Reducing Periodic Noise Using Soft Morphology Filter, Journal of Electronics (China), Vol. 21, No. 2, , pp. 159162.

[5] J. Yan, G. Lu and H. Lu. 2001 A SAR Image Enhancement Technique Based on Morphological Wavelet Transformation, Proceedings of International Conference on Multispectral and Hyperspectral Image Acquisition and Processing, Wuhan, China, pp. 203-208.

[6] J-N. Chi, D-S. Wang, Y. Duan and X-H. Xu. 2005 Algorithm of Image Enhancement Based on Order Morphology Filtering and Image Entropy Difference, Proceedings of the Fourth International Conference on Machine Learning and Cybernetics, Guangzhou, pp. $5105-5110$

[7] M. Seul, L. O'Gorman and M.J. Sammon, 2000 Practical Algorithms for Image Analysis. Cambridge University Press.
[8] M. Wirth and J. Lyon, 2005 Selective image enhancement using attribute morphology, Journal Imaging Science, Vol. 53, No. 4, Maney Publishing, pp. 195-198.

[9] Nursuriati Jamil \& Tengku Mohd Tengku Sembok. 2003. Gradient-Based Edge Detection of Songket Motifs. Digital Libraries: Technology and Management of Indigeneous Knowledge, pp. 456-467, Springer-Verlag Berlin.

[10] Nursuriati Jamil, Zainab Abu Bakar \& Tengku Mohd Tengku Sembok. 2004 A Comparison of Noise Removal Techniques in Songket Motif Images. IEEE Computer Society Conference on Computer Graphics, Image and Visualization (CGIV '04), pp. 39-143.

[11] P. Soille, 2002 Morphological Image Analysis, Springer Verlag, Berlin.

[12] R.A. Peters, 1995. A New Algorithm for Image Noise Reduction Using Mathematical Morphology, IEEE Trans. on Image Processing, Vol. 4, No.5, pp. 554-568.

[13] S. Tsekeridou, C. Kotropoulos and I. Pitas, 1996 Morphological Signal Adaptive Median Filter for Noise Removal, International Conference on Electronics, Circuits and Systems (ICECS 96), Rhodes, Greece, pp. 191-194.

[14] S. Umbaugh, 1999 Computer Vision and Image Processing. Prentice Hall Inc.

[15] www.mathswork.com (http://in.mathworks.com/help/images/structuringelements.html) 